\title{
Human bronchial epithelium orchestrates dendritic cell activation in severe asthma
}

\author{
Delphine Gras ${ }^{1}$, Asuncion Martinez-Anton ${ }^{1}$, Arnaud Bourdin ${ }^{2}$, Celine Garulli ${ }^{1}$, \\ Laure de Senneville ${ }^{1}$, Isabelle Vachier ${ }^{2}$, Joana Vitte ${ }^{1,3,4}$ and Pascal Chanez ${ }^{1,3,5}$
}

Affiliations: ${ }^{1}$ UMR INSERM 1067/CNRS 7333, Marseille, France. ${ }^{2}$ Departement des Maladies respiratoires, CHU Montpellier and PhyMedExp, Université de Montpellier, INSERM U1046, CNRS UMR 9214, Hôpital Arnaud de Villeneuve, Montpellier, France. ${ }^{3}$ Aix-Marseille Université, Marseille, France. ${ }^{4}$ APHM (Assistance Publique Hôpitaux de Marseille), Laboratoire d'Immunologie, Hôpital de la Conception, Marseille, France. ${ }^{5}$ APHM, Clinique des bronches, de l'allergie et du Sommeil, Hôpital Nord, Marseille, France.

Correspondence: Delphine Gras, UMR INSERM U1067 CNRS 7333 - Laboratoire d'Immunologie, Hôpital de la Conception, Pavillon Cornil, Marseille 13005, France. E-mail: delphine.grasQuniv-amu.fr

@ERSpublications

Normal or asthmatic status of bronchial epithelium shapes the function of the epithelial-dendritic cell trophic unit http://ow.ly/teQ43085KRf

Cite this article as: Gras D, Martinez-Anton A, Bourdin A, et al. Human bronchial epithelium orchestrates dendritic cell activation in severe asthma. Eur Respir J 2017; 49: 1602399 [https://doi.org/10.1183/ 13993003.02399-2016].

ABSTRACT The innate immune response is impaired in asthma, with increased epithelial release of C-X-C motif chemokine ligand (CXCL)8, interleukin (IL)-33 and thymic stromal lymphopoietin (TSLP). We hypothesised that dendritic cells might modulate the hyperresponsive epithelium in severe asthma.

For this purpose, we investigated epithelial-dendritic crosstalk in normal and diseased conditions, and because ultrafine particulate matter may affect asthmatic airways, we investigated its impact on this crosstalk. Air-liquid interface cultures of human bronchial epithelial cells (HBEC) of control subjects (cHBEC) or severe asthma patients (saHBEC) were co-cultured with monocyte-derived dendritic cells (moDC).

Increased release of CXCL8, TSLP and IL-33 from saHBEC contrasted with CHBEC producing CXCL10 and CCL2. Regarding moDC activation, saHBEC co-cultures induced only upregulation of CD86 expression, while cHBEC yielded full moDC maturation with HLA-DR, CD80, CD86 and CD40 upregulation. Particulate matter stimulation of HBEC had no effect on cHBEC but stimulated CXCL8 and IL-33 release in saHBEC. Particulate matter impaired epithelium signalling (TSLP, IL-33 and CXCL8) in saHBEC co-cultures despite C-C chemokine ligand 2 induction.

Crosstalk between HBEC and moDC can be established in vitro, driving a T1-type response with cHBEC and a T2-type response with saHBEC. Normal or asthmatic status of HBEC differentially shapes the epithelial-dendritic responses. We conclude that control moDC cannot rescue the hyperresponsive airway epithelium of severe asthmatics.

This article has supplementary material available from erj.ersjournals.com

Received: May 232016 | Accepted after revision: Jan 032017

Clinical trial: The blood samples used in this study were part of a clinical trial registered at www.clinicaltrials.gov with identifier number NCT00793676.

Support statement: This work was supported by Société Française d'Allergologie, Fond de Recherche en Santé Respiratoire, ARARD and ANR. Funding information for this article has been deposited with the Crossref Funder Registry.

Conflict of interest: Disclosures can be found alongside this article at erj.ersjournals.com

Copyright OERS 2017 


\section{Introduction}

The paradigm of asthma immunopathogenesis relies on both innate and adaptive immune responses. Our current view places the epithelium as the main orchestrator of the immune response. Indeed, the airway epithelium provides physical and chemical protection against deleterious environmental agents in cooperation with the adjacent network of immune cells [1, 2], eliciting innate pathways through interleukin (IL)-25, IL-33 and thymic stromal lymphopoietin (TSLP) release, potentially amplified by specific antigen stimulation [3]. Dendritic cells, neutrophils and type 2 innate lymphoid cells (ILC2) have been shown to be targets for all these cytokines [4-7]. Moreover, evidence of neutrophil participation in the severe form of the disease supports consideration of C-X-C motif chemokine ligand (CXCL) 8 as another significant severe asthma partner $[8,9]$.

The bronchial epithelium and sentinel dendritic cells are at the front line against inhaled agents. Together, they must detect potentially noxious agents, and "decide" on whether to mount and orient an immune response [10-13]. In asthma, disruption of the airway epithelium integrity, the local inflammatory milieu and increased influx of activated immune cells pave the way for acute exacerbations and persistence of poor therapeutic control $[14,15]$. Moreover, cytokines associated with asthma exacerbations, as well as IL-4, IL-13 and type 1 interferon (IFN), have been shown to act on bone marrow release of C-C chemokine receptor (CCR) $2^{+}$precursors of lung myeloid cells, including dendritic cells, leading to alternative activation and T2 polarised immunity $[16,17]$. Interestingly, airway epithelial cells may play a role, in some instances, as antigen-presenting cells (APC) during the development of airway inflammatory and immune responses, notably via programmed death ligand (PD-L)1 (B7H1) and PD-L2 (B7DC) expression [18]. These two proteins are ligands of the T-lymphocyte receptor, and have been shown to take part in the pathogenesis of asthma, allergy and autoimmune diseases [19].

Both initiation and exacerbation of allergic asthma have been related to air pollution levels [20, 21], and environmental factors such as ambient particulate matter might impact the epithelial-immune cell crosstalk. Particles $<10 \mu \mathrm{m}$ in diameter enter the lower airways, affect local homeostasis and may elicit immune responses. Dendritic cells play a crucial role in sampling airway particles and locally initiating either immune response or tolerance $[1,12,22]$. Particulate matter trafficking across the airway epithelium may result in airway dendritic cell maturation through a mandatory epithelial activation step [23, 24]. In asthma, pulmonary dendritic cells perform antigen presentation locally, thus playing a major role during outbreaks of local inflammation [12]. Dendritic cell maturation requires TSLP receptor activation. However, most of these data are derived from mouse models of asthma, mostly using ovalbumin and viral stimulations. Little is known about impaired epithelium-dendritic cell crosstalk in humans and even less in severe asthma patients.

In this study, the complex interplay of airway epithelial and immune cells was modelled by means of a co-culture system, which allowed the study of intercellular communication and close monitoring of cell responses to particulate matter exposure in severe asthma patients. For this purpose, we co-cultured monocyte-derived dendritic cells (moDC) with fully differentiated airway epithelium of control or severe asthma patients cultured at an air-liquid interface (ALI) [25]. We show that bronchial epithelia derived from severe asthmatics and control subjects drive distinct chemokine and moDC phenotype profiles, and that particulate matter exposure further modulates these distinct responses. In addition, these results suggest that our human co-culture model may be of particular interest for further studies on asthma-related abnormalities of the epithelial-dendritic unit and the impact of inhaled compounds.

\section{Materials and methods}

Additional details are provided in the supplementary material.

\section{Study subjects}

Asthmatic patients $(n=15)$ and control subjects $(n=6)$ were recruited for endobronchial biopsies at the Pulmonology Department, Assistance Publique Hôpitaux de Marseille, Marseille, France. The study protocol was approved by the local ethics committee and was part of the Bronchial Obstruction and Asthma Cohort (COBRA (Cohorte Obstruction Bronchique et Asthme)) sponsored by the French National Institute of Health and Medical Research (IDRGB 2008-A00284-51, Afssaps 2008-0113). All subjects were informed about the nature and purpose of the study, and provided written consent before enrolment. Demographic characteristics are reported in table 1.

Peripheral blood monocytes were obtained from 13 control volunteers and eight severe asthmatics, who were sex and age matched with the bronchial biopsy patients. This study protocol was approved by the local ethic committee and was part of a clinical trial registered at www.clinicaltrials.gov with identifier number NCT00793676. Demographic characteristics of these subjects are reported in table 2. 
TABLE 1 Patients characteristics (endobronchial biopsies)

\begin{tabular}{lccc} 
& Controls & Severe asthmatics & p-value \\
\hline Subjects $\mathbf{n}$ & 6 & 15 & \\
Age years & $40(26-64)$ & $65(33-81)$ & $\mathrm{NS}$ \\
Females & $50 \%$ & $70 \%$ & $\mathrm{NS}$ \\
Asthma control & $\mathrm{NA}$ & $22 \%$ & $\mathrm{NA}$ \\
Exacerbations in the last year & $\mathrm{NA}$ & $2.5(0-12)$ & $\mathrm{NA}$ \\
Best FEV $\%$ predicted & $100(98-105)$ & $82.5(64-100)$ & 0.004 \\
Blood eosinophils per mm $^{3}$ & $160(130-220)$ & $100(40-600)$ & $\mathrm{NS}$ \\
Atopy & $0 \%$ & $50 \%$ & 0.04 \\
Sinusitis & $0 \%$ & $70 \%$ & 0.006 \\
GORD & $0 \%$ & $60 \%$ & 0.016 \\
At least one comorbid condition" & $16.7 \%$ & $66.7 \%$ & $\mathrm{NS}$ \\
Long-term OCS & $0 \%$ & $70 \%$ & 0.006
\end{tabular}

Data are presented as median (range) unless otherwise stated. FEV1: forced expiratory volume in $1 \mathrm{~s}_{\text {: }}$ GORD: gastro-oesophageal reflux disease; OCS: oral corticosteroids; NA: not applicable; Ns: nonsignificant.

\#: based on positive skin test; " ${ }^{\text {: }}$ systemic hypertension, osteoporosis, sleep apnoea syndrome or obesity.

Human bronchial epithelial cell primary culture in ALI

Control or severe asthmatic human bronchial epithelial cells (cHBEC and saHBEC, respectively) were obtained from bronchial biopsy and cultured under ALI conditions as previously described [25].

Monocyte-derived dendritic cells

moDC were generated through in vitro differentiation of peripheral blood monocytes as previously described [26].

Particulate matter

The particulate matter consisted of endotoxin-free jet exhaust collected during experimental take-off/ landing cycles on an aero-engine bench as previously described [22].

Co-culture of bronchial epithelial cells and dendritic cells

On the sixth day, moDC were harvested, counted, centrifuged and resuspended in HBEC medium. cHBEC were co-cultured with control moDC and saHBEC were co-cultured with control or severe asthmatic moDC. The experimental design is illustrated in figure S1.

Immunoassays for epithelial and dendritic cells markers

Cytokines and chemokines measurement in basal supernatants

Levels of CCL2, CXCL10 and CXCL8 were measured with Cytometric Bead Array Flex Sets (Becton Dickinson, Le Pont de Claix, France) following the manufacturer's instructions. Levels of TSLP, IL-33 and IL25 were measured by ELISA (R\&D Systems, Lille, France).

moDC phenotype

After co-culture experiment, moDC were stained with specific antibodies directed against plasma membrane molecules involved in antigen presentation and costimulatory signal delivery (i.e. HLA-DR, CD40, CD80 and CD86 or control isotype-matched antibodies (Beckman Coulter, Villepinte, France)) for 30 min in the dark at room temperature $\left(18-25^{\circ} \mathrm{C}\right)$. Then, moDC were rinsed, resuspended in PBS containing $1 \%$ formaldehyde and analysed with an Epics XL (Beckman Coulter) flow cytometer. Data are presented as the median fluorescence intensity of the whole moDC population after isotype control subtraction.

\begin{tabular}{lccc}
\hline TABLE 2 Patients characteristics (blood samples) & & \\
& Controls & Severe asthmatics & p-value \\
\hline Subjects $\mathbf{n}$ & 13 & 8 & NS \\
Age years & $35(26-60)$ & $49(30-63)$ & NS \\
Females & $58 \%$ & $55 \%$ &
\end{tabular}

Data are presented as median (range) unless otherwise stated. Ns: nonsignificant. 
Real-time quantitative PCR analysis

Total RNA was purified from epithelial cells (RNeasy; Qiagen, Valencia, CA, USA) and cDNA was synthesised (Ready-to-Go RT-PCR beads; Amersham, Piscataway, NJ, USA). Human IL-25, PD-L1 and PD-L2 gene expression was determined by real-time quantitative PCR (LightCycler480; Roche, Basel, Switzerland) using SybrGreen, specific primers (Eurofins MWG Operon, Ebersberg, Germany) and $18 \mathrm{~S}$ rRNA as internal control.

\section{Results}

Effect of HBEC-moDC co-culture on HBEC mediator release and moDC phenotype

Control- and asthmatic-derived HBEC established confluent layers with a mucociliary phenotype, as previously described [25], with positive staining for mucin 5AC, $\beta$-tubulin IV and zonula occludens- 1 (figure S2A and B). Co-culture of HBEC with moDC did not alter viability ( $>98 \%$ by trypan blue exclusion) of either cell type after $24 \mathrm{~h}$. We ruled out the persistence of lymphocytes, and notably T-cells, in the moDC population through CD3 and CD19 staining (figure S2).

No cytokine or chemokine secretion was detectable in the supernatant from moDC in the absence of HBEC co-culture except for CCL2 (figure 1a-e).

CXCL10 and CCL2 production was significantly upregulated in CHBEC co-cultured with control moDC $(\mathrm{n}=6)$ compared to HBEC alone. Conversely, CXCL8, TSLP and IL-33 production was significantly upregulated in saHBEC co-cultured with control $(n=7)$ or asthmatic $(n=8)$ moDC compared to baseline (figure 1a-e and table S1). IL-25 secretion was undetectable. However, IL-25 mRNA expression was investigated and no difference was found (figure 1f).

Co-culture with moDC resulted in increased PD-L1 mRNA levels exclusively in saHBEC while PD-L2 mRNA increased in both cHBEC and saHBEC (figure $1 \mathrm{~g}$ and $\mathrm{h}$ ).

Expression of CD11c and CD83 was not altered by co-culture with either type of epithelium (figure 2a and b, respectively). However, the expression of HLA-DR, CD40, CD80 and CD86 was upregulated in control moDC co-cultured with cHBEC (figure 2c-f). Isolated upregulation of CD86 was found in control and asthmatic moDC co-cultured with saHBEC (figure $2 \mathrm{f}$ ).

The variation rate between the different clinical groups was compared for each studied marker (table S1). Only CD80 modulation was significantly different between the control moDC/cHBEC and both the control moDC/saHBEC and severe asthma moDC/saHBEC groups.

\section{Effect of particulate matter on HBEC mediator release}

Particulate matter examined by transmission electron microscopy (TEM) consisted of spherical primary particles with a mean diameter of $10 \mathrm{~nm}$ that formed small aggregates (figure 3a). Particulate matter laid on top of the HBEC layer was internalised by epithelial cells after 30 min to $2 \mathrm{~h}$ (figure $3 \mathrm{~b}$ and $\mathrm{c}$ ) and was found in HBEC cytoplasm at $24 \mathrm{~h}$ (figure $3 \mathrm{~d}$ ).

Basolateral production of CXCL8, CXCL10, CCL2, TSLP, IL-33 and IL-25 was measured in cHBEC ( $\mathrm{n}=6)$ and saHBEC $(n=15)$ cultures at baseline and in the presence of particulate matter. Particulate matter addition did not alter cHBEC responses compared to baseline levels but it resulted in increased CXCL8 and IL-33 release in saHBEC (figure 3e-i). As previously, IL-25 secretion was undetectable; therefore, we investigated IL-25 mRNA expression and we found a particulate matter-induced decrease in cHBEC (figure $3 \mathrm{j}$ ). This variation was significantly different compared to saHBEC group (table S2). Transcripts of the costimulatory molecules PD-L1 and PD-L2 were detectable at low and similar levels in cHBEC and saHBEC, and particulate matter addition for $24 \mathrm{~h}$ on the apical surface of HBEC did not induce any changes (figure $3 \mathrm{k}$ ).

\section{Effect of particulate matter on HBEC-moDC co-culture}

Particulate matter exposure of cHBEC co-cultured with moDC $(n=6)$ did not modify HBEC responses or moDC plasma membrane expression of molecules of interest previously described in this co-culture system. Particulate matter exposure of saHBEC co-cultured with control moDC $(n=7)$ induced an additional increase in basolateral production of CCL2, which was not found in saHBEC co-cultured with severe moDC. However, no further changes in moDC plasma membrane expression were found (figure 4 and table S3).

\section{Effect of conditioned moDC on lymphocytes}

IL-2, IL-4 and IL-6 were found to be increased, while IFN $\gamma$ and IL-10 were decreased in co-culture of saHBEC-conditioned moDC with lymphocytes compared to cHBEC-conditioned moDC/lymphocytes (figure 5). 

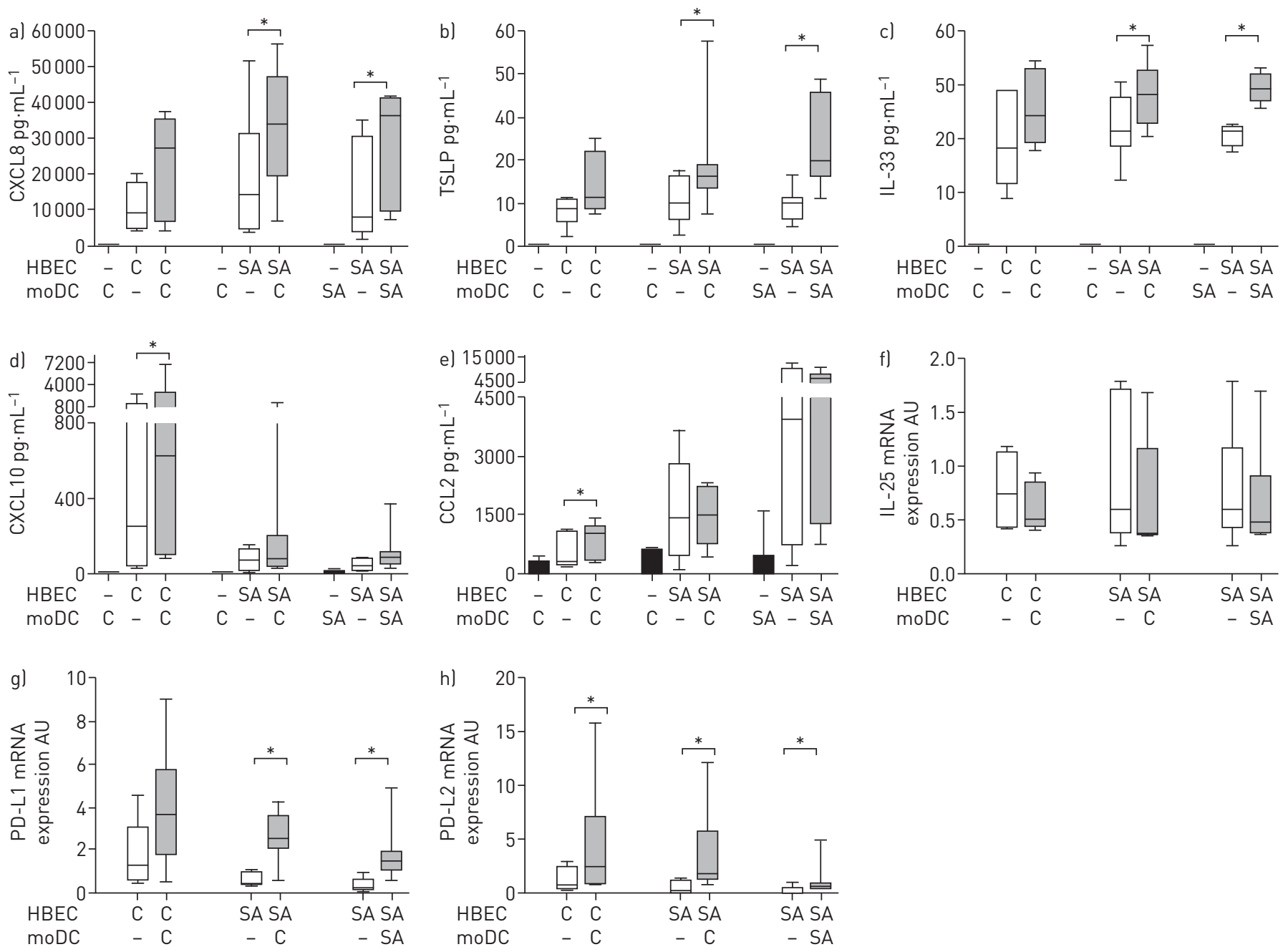

FIGURE 1 Effect of human bronchial epithelial cell (HBEC)-monocyte-derived dendritic cell (moDC) co-culture on chemokine/cytokine secretion and costimulatory molecule mRNA expression. HBECs from control subjects (C) or severe asthmatic patients (SA) were incubated for $24 \mathrm{~h}$ with moDC placed in the basolateral compartment. Levels of a) C-X-C motif chemokine ligand (CXCL)8, b) thymic stromal lymphopoietin (TSLP), c) interleukin (IL)-33, d) CXCL10 and e) C-C chemokine ligand (CCL)2 were measured in basolateral samples from air-liquid interface cultures. Semiquantitative analysis of f) IL-25, gl programmed death ligand (PD-L)1 and h) PD-L2 mRNA expression with 18S rRNA as a reporter gene. Horizontal lines represent the median, boxes represent the interquartile range and whiskers represent the range. ${ }^{*}$ : p<0.05, Wilcoxon test.

\section{Discussion}

Taking advantage of the reconstituted human ALI epithelium model [25], we set out to investigate further the epithelial-dendritic cell crosstalk in patients with severe asthma. Because ultrafine particulate matter may affect asthmatic airways, we questioned its impact on this crosstalk. First, we showed that epithelial-dendritic cell co-cultures upregulated the production of TSLP, IL-33 and CXCL8, epithelial PD-L1 and PD-L2 transcription, and moDC CD86 upregulation when epithelium was derived from severe asthmatic patients and irrespective of the origin of moDC. Moreover, particulate matter exposure of epithelia alone resulted in elevated CXCL8 and IL-33 production from asthmatic-derived epithelia, while leaving unchanged those derived from nonasthmatics, except for a decrease on IL-25 mRNA expression. This finding contrasted with responses of HBEC-moDC co-cultures whose HBEC originated from control donors, where upregulation of CCL2 and CXCL10 secretion, selective PD-L2 mRNA increase in HBEC, and a broader range of moDC presentation and costimulatory molecule upregulation (MHC class II, CD40, CD80 and CD86) were observed. By contrast, in severe asthmatics, addition of PM failed to modify previous responses on HBEC-moDC crosstalk, except for a CCL2 increase. Taken together, our results lend support to the role of HBEC as key orchestrators of the airway interface responses. Asthmatic or nonasthmatic status impacts on the HBEC-dendritic cell functional unit interaction with airborne (particular matter) and/or tissue (dendritic cell) players.

The majority of published studies use cell lines cultured in submersion for the study of the interaction with other cell types or the effect of particulate matter [27-30]. The most important strength of our study 

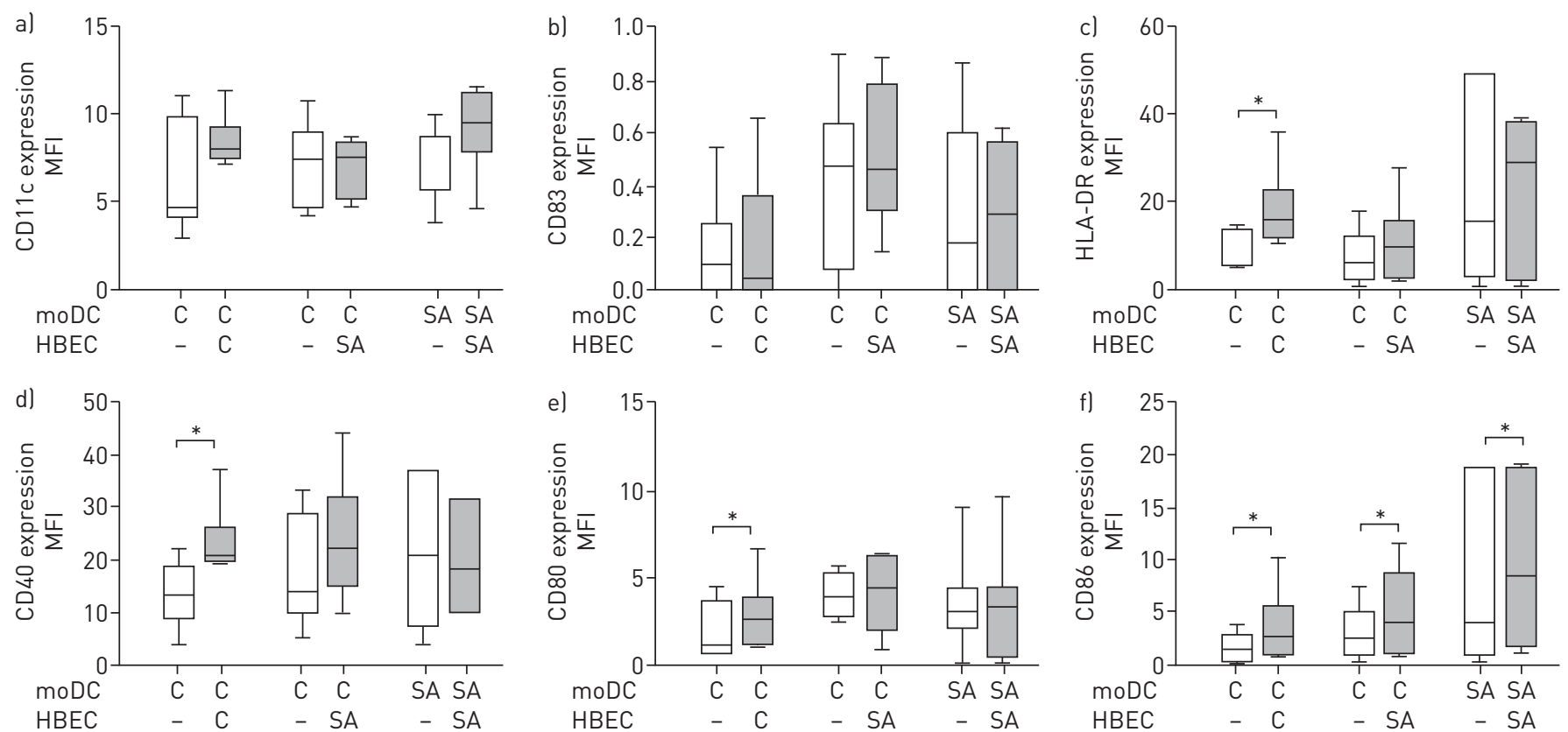

FIGURE 2 Monocyte-derived dendritic cell (moDC) phenotype after co-culture with human bronchial epithelial cells (HBEC). moDC from control or asthmatic subjects were incubated with HBEC from control (C) or severe asthmatic patients (SA) for $24 \mathrm{~h}$. Surface expression of a) CD11c, b) CD83, c) HLA-DR, d) CD40, e) CD80 and f) CD86 was measured by flow cytometry. Data are presented as the median fluorescence intensity (MFI) of the whole dendritic cell population after isotype control subtraction. Horizontal lines represent the median, boxes represent the interquartile range and whiskers represent the range. ${ }^{*}: p<0.05$, Wilcoxon test.

is the exclusive use of human primary cells obtained from patients, the ALI culture model allowing reconstitution of a completely differentiated epithelium, retaining the donor's epithelial secretory features [25], and human moDC. Blood monocytes and immature dendritic cells are recruited to the lung, predominantly under proinflammatory conditions but also for the steady-state turnover of pulmonary dendritic cells $[1,16,31]$. In vitro-derived moDC are phenotypically and functionally related to conventional (myeloid) dendritic cell found in inflamed airways [31-33].

Chemokine and cytokine patterns of secretion are of paramount importance for the HBEC-dendritic cell crosstalk, and are finely tuned by environmental and intrinsic properties of both the epithelium and immune cells. We showed in this study that saHBEC were more prone to alter their cytokine and chemokine pattern in the presence of particulate matter, with changes consisting of increased CXCL8 and IL-33. Involvement of CXCL8 in chronic respiratory diseases is well described and increased CXCL8 expression in the epithelium and subepithelium of the airway wall correlates with a decrease in lung function [34]. Excess airway CXCL8 may preclude normal epithelial responses to bacterial pathogens, through desensitisation of epithelial recognition under conditions of chronically elevated CXCL8 [35]. IL-33, a ligand of the ST2 receptor, differs from other IL-1 family members by its ability to promote Th2-type responses [36, 37]. Epithelial cells, which express both IL-33 and its receptor ST2, respond to IL-33 with CXCL8 upregulation [38]. Moreover, others cells, such as CD4 ${ }^{+}$T-lymphocytes or ILC2, also express ST2 and respond to IL-33, providing a direct link with adaptive immunity. A genome-wide association study identified the IL-33/ST2 axis as a potential key player in asthma, and a critical T2 driver through ILC2 recruitment and activation, irrespective of an allergen-specific stimulation. Our results suggest the persistence of this activated pathway in asthma at steady state and after particulate matter stimulation despite the presence of control or asthmatic moDC.

Moreover, we demonstrated that the co-culture of cHBEC with control moDC led to increased CCL2 and CXCL10 production. CCL2 released by HBEC is a strong chemoattractant of monocytes via its receptor CCR2 [39]. It acts on activated T-cells, natural killer cells and basophils [40, 41] similarly to CXCL10. CCL2 and CXCL10 are considered to be part of the Th1-type responses, including those elicited by respiratory viruses $[16,42]$. In contrast, in saHBEC-moDC coculture, an increase in CXCL8 and IL-33, as well as TSLP secretion, was observed. TSLP is a member of the hematopoietic cytokine family acting on dendritic cells, monocytes and T-cells [43]. TSLP expression is enhanced in asthmatic patients' airways and correlates with symptom scores [44]. TSLP acts on immature conventional dendritic cells, orienting them towards a pro-inflammatory T2 response [45]. CXCL8 has been shown to decrease the ability of 

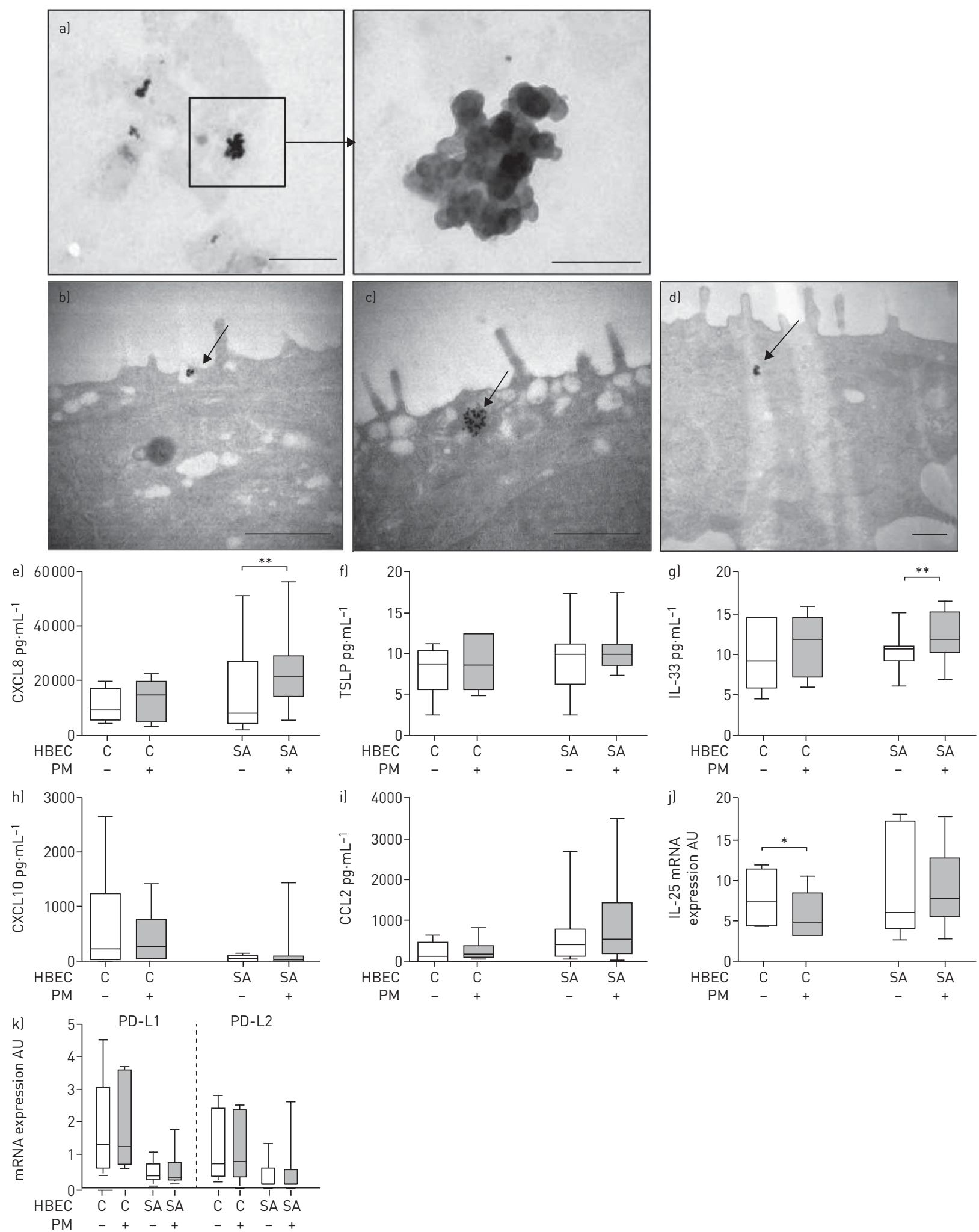

FIGURE 3 Effect of particulate matter (PM) addition on chemokine/cytokine secretion and costimulatory molecule mRNA expression. a) Representative micrograph of PM observed by transmission electron microscopy. Scale bars: low magnification, $1 \mu \mathrm{m}$; high magnification, 200 $\mathrm{nm}$. PM was incubated with human bronchial epithelial cells (HBEC) for b) 30 min (scale bar: $600 \mathrm{~nm}$ ), c) $2 \mathrm{~h}$ (scale bar: $600 \mathrm{~nm}$ ) or d) $24 \mathrm{~h}(0.5 \mu \mathrm{m}$ ). Arrows: PM uptake by HBEC. HBEC from control subjects (C) or severe asthmatic patients (SA) were incubated for $24 \mathrm{~h}$ with vehicle or PM added on the apical surface. Levels of e) C-X-C motif chemokine ligand (CXCL)8, f) thymic stromal lymphopoietin (TSLP), g) interleukin (IL)-33, h) CXCL10 and i) C-C chemokine ligand (CCL)2 were measured in basolateral samples from air-liquid interface cultures. Semiquantitative analysis of j) IL-25 mRNA and k) programmed death ligand (PD-L)1 and PD-L2 mRNA expression with 18S rRNA as a reporter gene. Horizontal lines represent the median, boxes represent the interquartile range and whiskers represent the range. ${ }^{*}$ : $p<0.05$, Wilcoxon test; ${ }^{* *}$ : $p<0.01$, Wilcoxon test. 

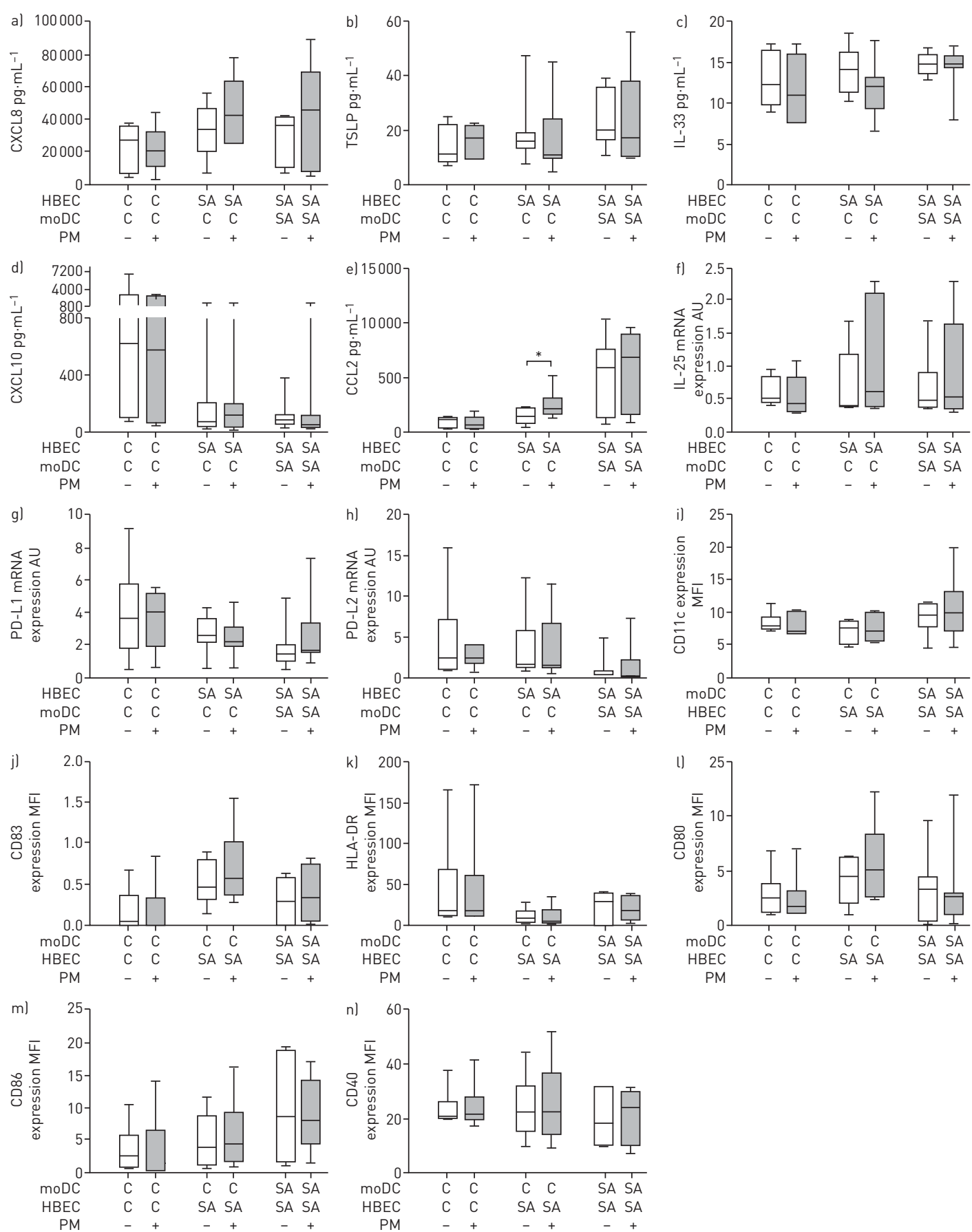

FIGURE 4 Effect of particulate matter (PM) addition on human bronchial epithelial cell (HBEC)-monocyte-derived dendritic cell (moDC) co-culture on chemokine/cytokine secretion, costimulatory molecule mRNA expression and moDC phenotype. HBEC from control subjects (C) or severe asthmatic patients (SA) were incubated for $24 \mathrm{~h}$ with moDC placed in basolateral compartment. PM was added concomitantly on the apical surface of the system. Levels of a) C-X-C motif chemokine ligand (CXCL)8, b) thymic stromal lymphopoietin (TSLP), c) interleukin (IL)-33, d) CXCL10 and e) C-C chemokine ligand (CCL)2 were measured in basolateral samples from air-liquid interface cultures. Semiquantitative analysis of f) IL-25, g) programmed death ligand (PD-L)1 and h) PD-L2 mRNA expression with 18S rRNA as a reporter gene. Surface expression of i) CD11c, j) CD83, k) HLA-DR, () CD80, m) CD86 and n) CD40 was measured by flow cytometry on control subject moDC incubated with HBEC from control or asthmatic patients. Results consist of the median fluorescence intensity (MFI) of the whole moDC population after isotypic control subtraction. Horizontal lines represent the median, boxes represent the interquartile range and whiskers represent the range. *: $p<0.05$, Wilcoxon test.

dendritic cells to prime naïve $\mathrm{T}$-cells and this effect was associated with incomplete upregulation of dendritic cells costimulatory molecules [46].

Interestingly, in some instances, airway epithelial cells can behave as APC during the development of airway inflammatory and immune responses, notably via PD-L1 and PD-L2 expression [18]. These 

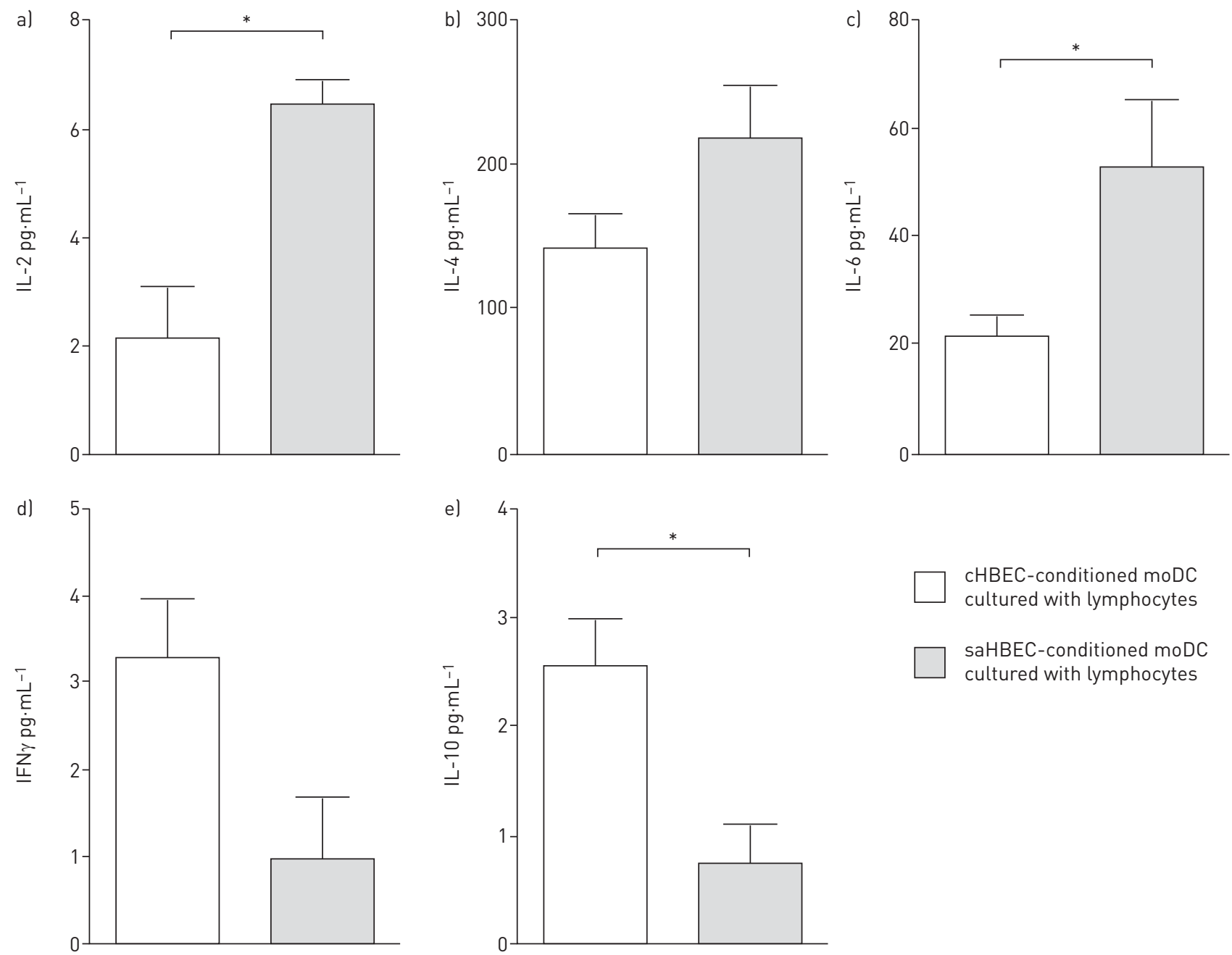

FIGURE 5 Effect of conditioned monocyte-derived dendritic cell (moDC)/lymphocyte co-culture on mediator release. moDC from control volunteers $(\mathrm{n}=3)$ were conditioned by incubation in supernatant from control (cHBEC) or severe asthma human bronchial epithelial cells (saHBEC) for $24 \mathrm{~h}$. After removing HBEC supernatants, moDC were cocultured with lymphocytes for an additional $48 \mathrm{~h}$. Levels of al interleukin (IL)-2, b) IL-4, c) IL-6, d) interferon (IFN) $\gamma$ and e) IL-10 were measured by cytometric bead array. Bars represent the mean and whiskers represent the standard deviation. ${ }^{*}$ : $p<0.05$, Mann-Whitney test.

molecules play grossly opposite roles [47], with PD-L1 engagement favouring the development of airway hyperreactivity and T2-type responses, while PD-L2 initiates T1-type responses and plays a protective role. Simultaneous expression of PD-L1 and PD-L2 neutralises their respective effects and does not lead to immediate polarisation of T-cells [19]. In our hands, moDC co-culture was associated with a simultaneous increase of PD-L1 and PD-L2 expression in saHBEC while PD-L2 alone increased in cHBEC. Taken together, these results suggest that the HBEC-dendritic cell functional unit promotes a defensive T1-type response in nonasthmatics, but displays an inappropriate mixed response in asthmatics, thus underscoring the importance of the epithelial status in further immune decision making.

moDC-HBEC co-cultures exposed to particulate matter showed no further modification with cHBEC, but displayed an increased CCL2 production with saHBEC. The lack of further CXCL10 or CCL2 upregulation in cHBEC-moDC co-cultures following particulate matter addition suggests a potent pro-T1 signal by itself. In contrast, CCL2 upregulation in saHBEC-moDC co-cultures suggests a monocyte chemotactic effect, and a self-sustaining recruitment and activation of inflammatory dendritic cell precursors.

Phenotypic modulation of dendritic cells is in line with the view of HBEC as instructors of dendritic cells towards a T1 polarising response through concomitant HLA-DR, CD40 and CD80 upregulation, and selective attenuation of allergen-induced IL-13, IL-5 and IL-9 $[32,48]$. cHBEC induced upregulation of HLA-DR, CD40, CD80 and CD86 expression on moDC, while saHBEC resulted in isolated upregulation of CD86. Thus, differential phenotypical modulation of dendritic cells was induced by asthmatic-versus 
nonasthmatic-derived epithelia; basically, dendritic cells exhibited signals suggesting incomplete maturation and antigen processing and presentation in asthma, despite active crosstalk illustrated by CCL2 expression.

In our study, HBEC and dendritic cells were derived from distinct donors, and this heterologous interaction may have impacted the functional responses of both cell types. We therefore checked the behaviour of autologous co-cultures and found that even though differences in the cytokine secretion levels existed between heterologous and autologous cultures, the trend of variation was the same (figures S3 and S4, and tables S4 and S5). The need for contact between HBEC and dendritic cells, notably through hypothetical dendritic cell digitations acting as sentinels within the epithelium, could not be addressed in our model because the insert pore diameter is $0.4 \mu \mathrm{m}$. Therefore, our study highlights that soluble mediators by themselves are able to induce a differential pattern of responses in asthmatics compared to healthy subjects, pointing out that contact is not always indispensable. Probably both direct and indirect interactions via soluble mediators are necessary to induce a complete response. In our model, we supposed that particulate matter may nonetheless have crossed the epithelium, either passively or actively. We also aimed to check if particulate matter were present in basolateral medium using TEM but culture medium-induced artefacts hampered the microscopic examination.

The route, dose and duration of corticosteroid treatment may affect epithelial behaviour, notably by a dual regulation of asthma-associated chemokines as mentioned by FUKAKUSA et al. [49]. However, using the ALI culture system, differentiated HBEC were used not earlier than 8 weeks after their last exposure to corticosteroids. While we did not directly assess the effect of corticosteroids in this study, characteristics of HBEC are considered as intrinsic and not due to their possible previous exposure to corticosteroids.

Our data support the view that nonasthmatic epithelium encourages effective T1-polarised responses, which are impaired in asthma due to epithelial and epithelium-induced abnormalities. To back this view, we performed some experiments $(n=3)$ of co-culture with HBEC-conditioned dendritic cells and lymphocytes. Cytokine secretion profiles of saHBEC-conditioned dendritic cells/lymphocytes showed a significant trend to differ with an increase of the T2-related response, i.e. IL-4 and IL-6, and a decrease of Th1-related response, i.e. IFN $\gamma$. Moreover, we showed a T-regulatory related response downregulation (IL-10 decrease) and self-sustained T-helper generation (IL2 increase).

In conclusion, we showed that crosstalk between human reconstituted bronchial epithelium and human moDC leads to a T1-polarised response in saHBEC, switching to a T2-type response in asthmatics, thus showing that epithelial phenotype alters dendritic cell responses. We present here a human co-culture model of the epithelial-dendritic unit of the bronchus that allows further dissection of the asthmatic versus nonasthmatic epithelial responses.

\section{Acknowledgements}

The authors are grateful to Daniel Ferry (CINaM, Marseille, France) for providing the particulate matter, Fabrice Richard and Jean-Paul Chauvin (IBDM, Marseille, France) for technical assistance with TEM and data analysis, Aurelie Fort (MedBioMed, Montpellier, France) for technical assistance in photo acquisition of epithelium and to Matthew Loza, Patrick Branigan and Frederic Baribaud (Johnson \& Johnson, Philadelphia, PA, USA) for English review of the manuscript.

\section{References}

1 Holt PG, Strickland DH, Wikstrom ME, et al. Regulation of immunological homeostasis in the respiratory tract. Nat Rev Immunol 2008; 8: 142-152.

Turvey SE, Broide DH. Innate immunity. J Allergy Clin Immunol 2010; 125: Suppl. 2, S24-S32.

Lambrecht BN, Hammad H. The airway epithelium in asthma. Nat Med 2012; 18: 684-692.

Hansel TT, Johnston SL, Openshaw PJ. Microbes and mucosal immune responses in asthma. Lancet 2013; 381: 861-873.

$5 \quad$ Holgate ST. Innate and adaptive immune responses in asthma. Nat Med 2012; 18: 673-683.

6 Kim BS, Siracusa MC, Saenz SA, et al. TSLP elicits IL-33-independent innate lymphoid cell responses to promote skin inflammation. Sci Transl Med 2013; 5: 170ra16.

7 Walker JA, Barlow JL, McKenzie AN. Innate lymphoid cells - how did we miss them? Nat Rev Immunol 2013; 13: 75-87.

8 Haldar P, Pavord ID. Noneosinophilic asthma: a distinct clinical and pathologic phenotype. J Allergy Clin Immunol 2007; 119: 1043-1052.

9 Nair P, Gaga M, Zervas E, et al. Safety and efficacy of a CXCR2 antagonist in patients with severe asthma and sputum neutrophils: a randomized, placebo-controlled clinical trial. Clin Exp Allergy 2012; 42: 1097-1103.

10 Barnes PJ. Immunology of asthma and chronic obstructive pulmonary disease. Nat Rev Immunol 2008; 8: 183-192.

11 Broide DH. Immunologic and inflammatory mechanisms that drive asthma progression to remodeling. J Allergy Clin Immunol 2008; 121: 560-570.

12 Gill MA. The role of dendritic cells in asthma. J Allergy Clin Immunol 2012; 129: 889-901.

13 Vareille M, Kieninger E, Edwards MR, et al. The airway epithelium: soldier in the fight against respiratory viruses. Clin Microbiol Rev 2011; 24: 210-229.

14 Chanez P. Severe asthma is an epithelial disease. Eur Respir J 2005; 25: 945-946.

15 Holgate ST. Epithelium dysfunction in asthma. J Allergy Clin Immunol 2007; 120: 1233-1244. 
16 Holt PG, Strickland DH, Sly PD. Virus infection and allergy in the development of asthma: what is the connection? Curr Opin Allergy Clin Immunol 2012; 12: 151-157.

17 Subrata LS, Bizzintino J, Mamessier E, et al. Interactions between innate antiviral and atopic immunoinflammatory pathways precipitate and sustain asthma exacerbations in children. J Immunol 2009; 183: 2793-2800.

18 Kim J, Myers AC, Chen L, et al. Constitutive and inducible expression of B7 family of ligands by human airway epithelial cells. Am J Respir Cell Mol Biol 2005; 33: 280-289.

19 Singh AK, Stock P, Akbari O. Role of PD-L1 and PD-L2 in allergic diseases and asthma. Allergy 2011; 66: $155-162$.

20 Downs SH, Schindler C, Liu LJ, et al. Reduced exposure to PM10 and attenuated age-related decline in lung function. N Engl J Med 2007; 357: 2338-2347.

21 McCreanor J, Cullinan P, Nieuwenhuijsen MJ, et al. Respiratory effects of exposure to diesel traffic in persons with asthma. N Engl J Med 2007; 357: 2348-2358.

22 Ferry D, Rolland C, Delhaye D, et al. Jet exhaust particles alter human dendritic cell maturation. Inflamm Res 2011; 60: 255-263.

23 Bleck B, Tse DB, Jaspers I, et al. Diesel exhaust particle-exposed human bronchial epithelial cells induce dendritic cell maturation. J Immunol 2006; 176: 7431-7437.

24 Pichavant $\mathrm{M}$, Taront $\mathrm{S}$, Jeannin $\mathrm{P}$, et al. Impact of bronchial epithelium on dendritic cell migration and function: modulation by the bacterial motif KpOmpA. J Immunol 2006; 177: 5912-5919.

25 Gras D, Bourdin A, Vachier I, et al. An ex vivo model of severe asthma using reconstituted human bronchial epithelium. J Allergy Clin Immunol 2012; 129: 1259-1266.

26 Nair S, Archer GE, Tedder TF. Isolation and generation of human dendritic cells. Curr Protoc Immunol 2012; Chapter 7: Unit 7.32.

27 Bleck B, Grunig G, Chiu A, et al. MicroRNA-375 regulation of thymic stromal lymphopoietin by diesel exhaust particles and ambient particulate matter in human bronchial epithelial cells. J Immunol 2013; 190: 3757-3763.

28 Li YJ, Shimizu T, Hirata Y, et al. EM, EM703 inhibit NF- $\kappa \mathrm{B}$ activation induced by oxidative stress from diesel exhaust particle in human bronchial epithelial cells: importance in IL-8 transcription. Pulm Pharmacol Ther 2013; 26: 318-324.

29 Matthews NC, Faith A, Pfeffer P, et al. Urban particulate matter suppresses priming of T helper type 1 cells by granulocyte/macrophage colony-stimulating factor-activated human dendritic cells. Am J Respir Cell Mol Biol 2014; 50: 281-291.

30 Ovrevik J, Refsnes M, Totlandsdal AI, et al. TACE/TGF- $\alpha$ /EGFR regulates CXCL8 in bronchial epithelial cells exposed to particulate matter components. Eur Respir J 2011; 38: 1189-1199.

31 Rate A, Upham JW, Bosco A, et al. Airway epithelial cells regulate the functional phenotype of locally differentiating dendritic cells: implications for the pathogenesis of infectious and allergic airway disease. J Immunol 2009; 182: 72-83.

32 Geissmann F, Manz MG, Jung S, et al. Development of monocytes, macrophages, and dendritic cells. Science 2010; 327: 656-661.

33 Shi C, Pamer EG. Monocyte recruitment during infection and inflammation. Nat Rev Immunol 2011; 11: 762-774.

34 Shannon J, Ernst P, Yamauchi Y, et al. Differences in airway cytokine profile in severe asthma compared to moderate asthma. Chest 2008; 133: 420-426.

35 Zhang T, Song KW, Hekmat-Nejad M, et al. A modeling-derived hypothesis on chronicity in respiratory diseases: desensitized pathogen recognition secondary to hyperactive IRAK/TRAF6 signaling. PLoS One 2009; 4: e5332.

36 Schmitz J, Owyang A, Oldham E, et al. IL-33, an interleukin-1-like cytokine that signals via the IL-1 receptor-related protein ST2 and induces T helper type 2-associated cytokines. Immunity 2005; 23: 479-490.

37 Su Z, Lin J, Lu F, et al. Potential autocrine regulation of interleukin-33/ST2 signaling of dendritic cells in allergic inflammation. Mucosal Immunol 2013; 6: 921-930.

38 Yagami A, Orihara K, Morita H, et al. IL-33 mediates inflammatory responses in human lung tissue cells. J Immunol 2010; 185: 5743-5750.

39 Ip WK, Wong CK, Lam CW. Interleukin (IL)-4 and IL-13 up-regulate monocyte chemoattractant protein-1 expression in human bronchial epithelial cells: involvement of p38 mitogen-activated protein kinase, extracellular signal-regulated kinase $1 / 2$ and Janus kinase-2 but not c-Jun NH2-terminal kinase $1 / 2$ signalling pathways. Clin Exp Immunol 2006; 145: 162-172.

40 Deshmane SL, Kremlev S, Amini S, et al. Monocyte chemoattractant protein-1 (MCP-1): an overview. J Interferon Cytokine Res 2009; 29: 313-326.

41 Korpi-Steiner NL, Valkenaar SM, Bates ME, et al. Human monocytic cells direct the robust release of CXCL10 by bronchial epithelial cells during rhinovirus infection. Clin Exp Allergy 2010; 40: 1203-1213.

42 Dougherty RH, Fahy JV. Acute exacerbations of asthma: epidemiology, biology and the exacerbation-prone phenotype. Clin Exp Allergy 2009; 39: 193-202.

43 Comeau MR, Ziegler SF. The influence of TSLP on the allergic response. Mucosal Immunol 3: 138-147.

44 Ying S, O'Connor B, Ratoff J, et al. Thymic stromal lymphopoietin expression is increased in asthmatic airways and correlates with expression of Th2-attracting chemokines and disease severity. J Immunol 2005; 174: 8183-8190.

45 Soumelis V, Reche PA, Kanzler $\mathrm{H}$, et al. Human epithelial cells trigger dendritic cell mediated allergic inflammation by producing TSLP. Nat Immunol 2002; 3: 673-680.

46 Yoshikawa T, Hill T, Li K, et al. Severe acute respiratory syndrome (SARS) coronavirus-induced lung epithelial cytokines exacerbate SARS pathogenesis by modulating intrinsic functions of monocyte-derived macrophages and dendritic cells. J Virol 2009; 83: 3039-3048.

47 Keir ME, Butte MJ, Freeman GJ, et al. PD-1 and its ligands in tolerance and immunity. Annu Rev Immunol 2008; 26: 677-704

48 Kuchroo VK, Das MP, Brown JA, et al. B7-1 and B7-2 costimulatory molecules activate differentially the Th1/Th2 developmental pathways: application to autoimmune disease therapy. Cell 1995; 80: 707-718.

49 Fukakusa M, Bergeron C, Tulic MK, et al. Oral corticosteroids decrease eosinophil and CC chemokine expression but increase neutrophil, IL-8, and IFN- $\gamma$-inducible protein 10 expression in asthmatic airway mucosa. $J$ Allergy Clin Immunol 2005; 115: 280-286. 